\section{Hallazgo de una nueva mutación en una familia chilena con diabetes monogénica. Caso clínico}

\author{
MARCOS ESTICA R. ${ }^{1}$, DANIELA SEELENFREUND H. ${ }^{2, a, b}$, \\ PILAR DURRUTY A. ${ }^{1,3, a}$, GLORIA BRIONES B. ${ }^{1}$
}

\section{Finding of a new mutation in a chilean family with monogenic diabetes. Report of one case}

We report a 21 years old woman, without offspring, with diabetes mellitus diagnosed at 17 years of age, without ketosis or weight loss. Her body mass index was $18 \mathrm{~kg} / \mathrm{m}^{2}$. Her C peptide was normal $(2.3 \mathrm{ng} / \mathrm{ml})$ and diabetes mellitus type 1 autoantibodies were negative. A monogenic diabetes Maturity Onset Diabetes of the Young (MODY) was proposed. Her family study disclosed a diabetic father and a brother with altered fasting glucose levels. The University of Exeter score for MODY yielded a 75.5\% probability of MODY2. In the genetic-molecular study of the glucokinase gene (MODY2), the patient had a mutation at position 1343 of exon 10, corresponding to a heterozygous substitution of guanine by adenine (1343 G>A). The same mutation was found in her father and brother. This mutation is different from those previously described in the literature. The described change determines that a glycine is replaced by aspartic at amino acid 448 of the enzyme (non-synonymous substitution). The diagnosis of MODY2 was therefore confirmed in the patient and her father. The mutation was inherited by paternal line.

(Rev Med Chile 2018; 146: 929-932)

Key words: Diabetes Mellitus, Type 2; Glucokinase; Mutation, Missense.
'Unidad de Diabetes. Hospital San Juan de Dios. Santiago, Chile. ${ }^{2}$ Departamento de Bioquímica y Biología Molecular, Facultad de Ciencias Químicas y Farmacéuticas, Universidad de Chile. Santiago, Chile.

${ }^{3}$ Sección Endocrinología y Diabetes. Hospital Clínico Universidad de Chile. Santiago,

Chile.

aBioquímico.

${ }^{\mathrm{b}} \mathrm{PhD}$

Este reporte de caso clínico fue sin apoyo financiero. Los autores declaran no tener conflictos de interés.

Recibido el 16 de marzo de 2018, aceptado el 6 de julio de 2018 .

Correspondencia a: Marcos Estica R.

Chacabuco 419, Santiago. marcosestica@gmail.com
E n Chile se utiliza la clasificación actual de Diabetes Mellitus (DM) de la American Diabetes Association (ADA) ${ }^{1}$. Se basa en la etiopatogenia de la enfermedad y considera 4 categorías: I. Diabetes mellitus tipo 1; II. Diabetes mellitus tipo 2; III. Diabetes gestacional y IV. Otros tipos específicos de diabetes; éstos en conjunto representan el 1\% de total de los casos de DM.

En la categoría IV se consideran los subtipos desde la A hasta la $\mathrm{H}$, y en la subcategoría A se incluyen las DM por defectos genéticos de la función de la célula beta (cel $\beta$ ). Entre ellas se conocen las diabetes monogénicas, que son producidas por mutaciones en los genes que se expresan en las cel $\beta$ y que determinan el deterioro en la síntesis o en la secreción de insulina ${ }^{2}$. Este subgrupo de
DM comprende los Maturity Onset Diabetes of the Young (MODY) que representa 1\% de los diabéticos en Europa. En 1987 la casuística de la Unidad de Diabetes del Hospital San Juan de Dios, informaba que el 0,46 del total de pacientes atendidos era MODY ${ }^{3}$.

Se denomina MODY al grupo de diabéticos que se diagnostica generalmente antes de los 25 años de edad ${ }^{2}$, cuyas características se heredan con patrón autosómico dominante y penetrancia incompleta. Estos pacientes habitualmente tienen un estado nutricional normal, ausencia de estigmas de Resistencia a la Insulina (RI), historia familiar de DM en tres generaciones. Sin embargo, pueden existir casos índices sin antecedentes de DM en la familia. 
Los MODY se clasifican de acuerdo a las presentaciones clínicas y según los diferentes genes afectados. Los más frecuentes son los MODY que afectan al gen que codifica la glucoquinasa (GCK) o MODY2 y los MODY por factores de transcripción (MODY1 y 3). En los MODY2 se afecta la respuesta secretora de insulina, presentando hiperglicemia de ayunas. La enfermedad no es progresiva, no requieren tratamiento farmacológico (excepto en el embarazo) y no se asocia a complicaciones crónicas ${ }^{4}$.

Frecuentemente los pacientes MODY se diagnostican como tipo 2 (DM2) y no reciben el tratamiento adecuado. Presentamos este caso clínico para orientar respecto a la pesquisa, el diagnóstico y el mejor manejo de este subtipo de DM.

\section{Caso clínico}

Mujer, de 21 años, sin hijos. A los 17 años se le diagnosticó DM por presentar polidipsia y poliuria, asociada a glicemia de ayunas $130 \mathrm{mg} / \mathrm{dl}$, confirmada con prueba de tolerancia a la glucosa oral (PTGO) de $75 \mathrm{~g}: 116 \mathrm{mg} / \mathrm{dl}$ ayunas y $234 \mathrm{mg} /$ dl post carga. Se descartó DM1 por autoanticuerpos negativos y péptido C normal, $\mathrm{HbAlc} 6,8 \%$ al ingreso y orina sin glucosuria. En el examen físico no se encontraron signos de RI, talla $1,56 \mathrm{~m}$ y peso $44,5 \mathrm{~kg}$, IMC $18 \mathrm{~kg} / \mathrm{m}^{2}$. Se indicó tratamiento no farmacológico y metformina $850 \mathrm{mg} / 2$ veces al día. Evolucionó satisfactoriamente durante 4 años, sin presentar complicaciones, mantuvo HbAlc entre 6-7\%. En enero de 2015 se suspendió la metformina por intolerancia gástrica y considerando la evolución y los antecedentes familiares de DM, se planteó que podría presentar MODY.

Se estudió la DM en familiares con PTGO, pesquisándose DM en el padre. Su hermano presentaba anormalidad de la glicemia. La madre no tenía DM (Tabla 1).

De la anamnesis familiar destaca una fuerte presencia de DM por la línea paterna, donde 8 de 9 hermanos del padre del caso índice, están diagnosticados con DM2, además de ambos abuelos. Por la línea materna, solamente la abuela y una tía tienen DM2.

Se utilizó la aplicación de la Universidad de Exeter ${ }^{5}$, estimándose que la paciente presenta MODY con una probabilidad de 75,5\%. Se decide realizar un estudio genético familiar, para lo cual se contaba con el consentimiento informado de los participantes y la aprobación del Comité de Ética del Hospital San Juan de Dios.

Se realizó un análisis genético-molecular (Laboratorio de Genética de la Red UC-Christus Santiago, Chile) de los exones 1 a 10 del gen GCK mediante secuenciación directa de las regiones codificantes y de las uniones intrón-exón. La paciente presentó una mutación en la posición 1343 en el exón 10 del gen $G C K$, correspondiente a un cambio de guanina por adenina ( $1343 \mathrm{G}>\mathrm{A}$ ); en el otro alelo no se encontró dicha mutación (paciente heterocigota).

El padre y el hermano presentan la misma mutación del gen GCK en forma heterocigota. La madre mostró ambos alelos silvestres, por lo cual no es MODY2 ni tiene DM (Tabla 1). La variante encontrada probablemente es patogénica, pues genera un cambio no sinónimo o missense del aminoácido (aa) glicina por ácido aspártico (Gly448Asp) en la enzima glucoquinasa (GCK). Estos resultados permiten establecer la presencia de MODY2 en una familia chilena debido a una mutación no descrita previamente.

Tabla 1. Parámetros clínicos, inmunológicos y genéticos de familia MODY2

\begin{tabular}{|lllll|}
\hline & Paciente índice & Padre & Madre & Hermano \\
\hline Glicemia venosa en ayunas & $130 \mathrm{mg} / \mathrm{dl}$ & $142 \mathrm{mg} / \mathrm{dl}$ & $97 \mathrm{mg} / \mathrm{dl}$ & $121 \mathrm{mg} / \mathrm{dl}$ \\
\hline Glicemia 2 h post carga $75 \mathrm{~g}$ & $234 \mathrm{mg} / \mathrm{dl}$ & $207 \mathrm{mg} / \mathrm{dl}$ & $125 \mathrm{mg} / \mathrm{dl}$ & $99 \mathrm{mg} / \mathrm{dl}$ \\
\hline Péptido C* & $2,3 \mathrm{ng} / \mathrm{ml}$ & $1,8 \mathrm{ng} / \mathrm{ml}$ & No aplica & $2,1 \mathrm{ng} / \mathrm{ml}$ \\
Autoanticuerpos para DM1 & Negativos & Negativos & No aplica & Negativos \\
Mutación glucoquinasa & Positiva variante & Positiva & Negativo para & Positiva \\
& Gli448Asp & variante Gli448Asp & mutación & variante Gli448Asp \\
\hline
\end{tabular}

*Rango normal: 1,1 - 4,4 ng/ml. 


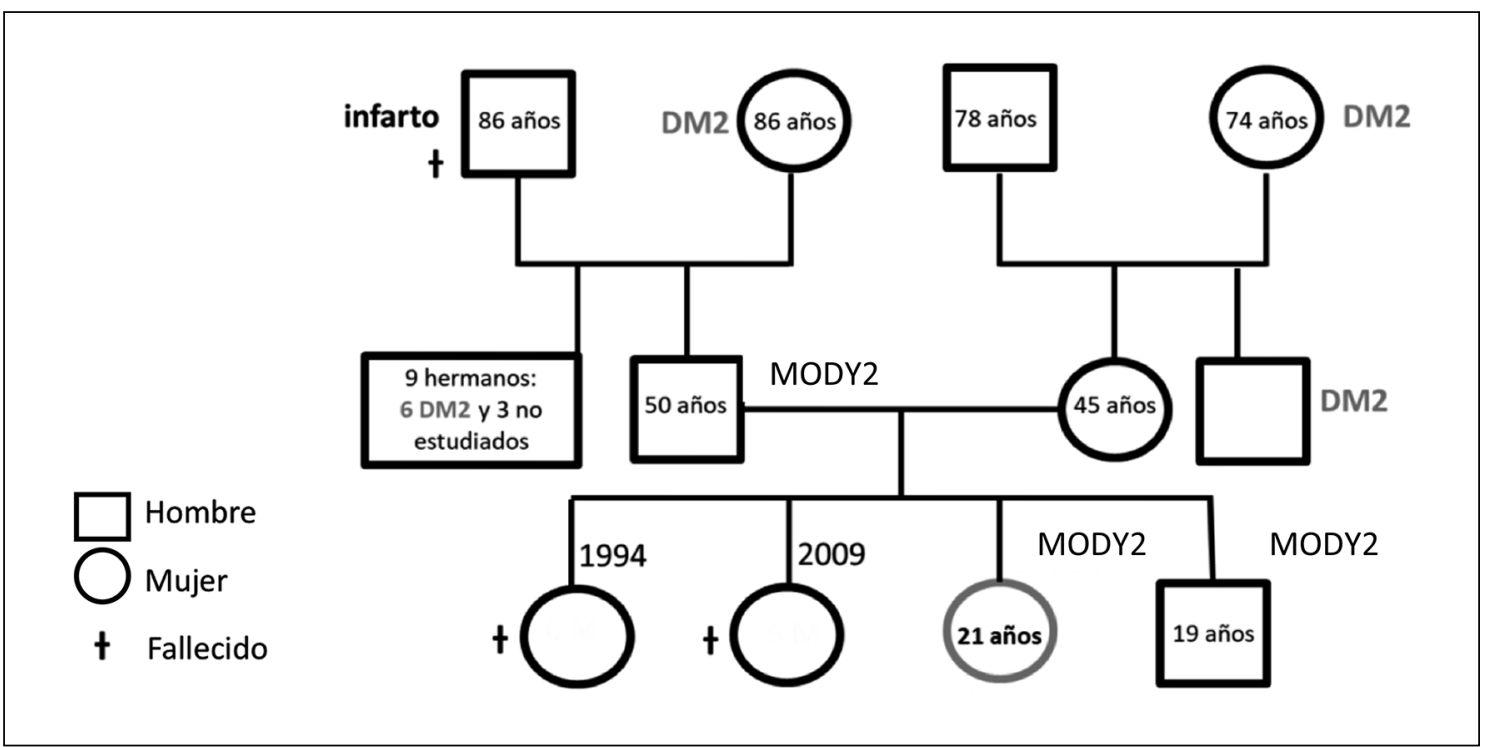

Figura 1. Patrón de herencia familiar de MODY2.

\section{Discusión}

El estudio del gen GCK permite identificar aproximadamente $30-50 \%$ de las mutaciones en pacientes con DM monogénica, dependiendo de la población investigada ${ }^{6}$ y hasta el $96,5 \%$ de los sujetos con $\mathrm{MODY}^{7}$. El estudio indica que la paciente y su padre presentan una DM monogénica con una mutación en el gen $G C K$, confirmando el diagnóstico de MODY2 (Figura 1).

El hermano tiene MODY2, aunque de acuerdo a sus parámetros clínicos se clasificaría como prediabético, se establece que la mutación es heredada del padre. La variación encontrada es distinta a las informadas en otros pacientes chilenos ${ }^{8,9}$.

El gen GCK (OMIM 138079) se ubica en el cromosoma 7 (7p13) y está formado por 10 exones y presenta tres variantes alternativas del exón 1 , cuyo procesamiento es tejido-específico, pues el exón la se expresa en las cél $\beta$ del páncreas y los exones $1 b$ y 1c en hígado ${ }^{10}$. A diferencia de lo descrito para otras patologías, las distintas mutaciones del gen GCK en todos los casos determinan MODY2. Cabe destacar que éstas no afectan la penetrancia, ni anticipación de la enfermedad. Es una condición dominante, pues se presenta en heterocigosis; sin embargo, se ha descrito que en homocigosis o como mutación compuesta (dos mutaciones diferentes en el mismo gen $G C K$ ) determinan el desarrollo de diabetes neonatal permanente ${ }^{11}$.

En la literatura se han descrito alrededor de 620 mutaciones en distintas poblaciones ${ }^{12,13}$ y más de 5.000 polimorfismos en GCK (www.ncbi.nlm. nih.gov/projects/SNP/snp_ref.cgi?geneId=2645). Se han informado diversas mutaciones no sinónimas en el exón 10; no obstante esta variante no ha sido encontrada ${ }^{13,14}$, aunque confirma la etiología de MODY2.

El gen CGK afectado codifica la enzima GCK que cataliza la primera etapa limitante de la glicolisis, correspondiente a la conversión de glucosa a glucosa-6-fosfato. Su función fisiológica es regular la entrada de ésta a las cel $\beta$ y hepatocitos, especialmente con glicemia alta $^{10}$. Se trata de una proteína monomérica y relativamente pequeña $(52 \mathrm{KDa})$, con extremos $\mathrm{N}$-terminal distintos determinados por su procesamiento tejido específico. Presenta una compleja regulación de tipo alostérica ${ }^{14}$. En las cel $\beta$ la GCK es regulada a nivel traduccional o postraduccional principalmente por glucosa, en cambio en el hepatocito la enzima es regulada primordialmente a nivel transcripcional por insulina ${ }^{10}$.

Es posible especular que el cambio de glicina (aa neutro) por ácido aspártico (aa con carga + ) en una región cercana al centro hidrofóbico y al único 
sitio de unión de la glucosa (sustrato) podría afectar la estructura, y por ende la funcionalidad de la enzima. Si la mutación descrita altera la actividad o regulación de la GCK, explicaría la patogenia de estos MODY2 en términos moleculares. A futuro, se podría estudiar esta mutación modelando la estructura de la enzima ya que probablemente afectaría la actividad de la GCK.

Resulta importante definir el subtipo de DM monogénica y descartar otros tipos de DM. Se recomienda realizar estudios genéticos precozmente cuando se sospecha un MODY, para indicar el adecuado tratamiento. En los exámenes, uno de los padres generalmente tendrá un nivel levemente elevado de la glicemia de ayunas, a menos que la mutación haya surgido de novo.

\section{Referencias}

1. Standards of Medical Care in Diabetes-2018. Classification and diagnosis of diabetes. Diabetes Care 2018; 41 (Suppl 1): S13-S27.

2. Sanzana MG, Durruty P. Otros tipos específicos de diabetes mellitus. Rev Med Clin (Las Condes) 2016; 27 : 160-70.

3. Durruty P, Muñoz S, García de los Ríos M. Frecuencia de diabetes mellitus y secreción beta pancreática en hijos de pacientes MODY. Rev Med Chile 1987; 115: 404-10.

4. Schields BM, Shepherd M, Hudson M, McDonald TJ, Coldough K, Peters J, et al. Population-Based Assessment of a Biomarker-Based Screening Pathway to Aid Diagnosis of Monogenic Diabetes in Young-Onset Patients. Diabetes Care 2017; 40: 1017-25.

5. Shields BM, McDonald TJ, Ellard S, Campbell MJ, Hyde C, Hattersley AT. The development and validation of a clinical prediction model to determine the probability of MODY in patients with young-onset diabetes. Diabetologia 2012; 55: 1265-72.
6. Yang Y, Chan L. Monogenic Diabetes: What it teaches us on the common forms of type 1 and type 2 Diabetes. Endocrine Reviews 2016; 37: 190-222.

7. Ellard S, Bellanné-Chantelot C, Hattersley AT. European Molecular Genetics Quality Network (EMQN) MODY group. Best practice guidelines for the molecular genetic diagnosis of maturity-onset diabetes of the young. Diabetologia 2008; 51: 546-53.

8. Codner E, Deng L, Pérez-Bravo F, Román R, Lanzano $\mathrm{P}$, Cassorla F, et al. Glucokinase mutations in young children with hyperglycemia. Diabetes Metab Res Rev 2006; 22: 348-55.

9. Pollak F, Lagos M, Santos J, Poggi, Helena, Urzúa A, et al. Diabetes mellitus por mutación en el gen de glucokinasa. Caso clínico. Rev Med Chile 2017; 145: 1203-7.

10. Matschinsky FM. Regulation of pancreatic beta-cell glucokinase: from basics to therapeutics. Diabetes 2002; 51 (Suppl 3): S394-404.

11. Owen KR, McCarthy MI. Genetics of type 2 diabetes. Curr Opin Genet Dev 2007; 17: 239-44.

12. Gloyn AL. Glucokinase (GCK) mutations in hyper- and hypoglycemia: maturity-onset diabetes of the young, permanent neonatal diabetes, and hyperinsulinemia of infancy. Hum Mutat 2003; 22: 353-62.

13. Osbak KK, Colclough K, Saint-Martin C, Beer NL, Bellanné-Chantelot C, Ellard S, et al. Update on mutations in glucokinase (GCK), which cause maturity-onset diabetes of the young, permanent neonatal diabetes, and hyperinsulinemic hypoglycemia. Hum Mutat 2009; 30: 1512-26.

14. Vits L, Beckers D, Craen M, de Beaufort C, Vanfleteren E, Dahan K, et al. Identification of novel and recurrent glucokinase mutations in Belgian and Luxembourg maturity onset diabetes of the young patients. Clin Genet 2006; 70: 355-9.

15. Whittington AC, Larion M, Bowler JM, Ramsey KM, Brüschweiler R, Miller BG. Dual allosteric activation mechanisms in monomeric human glucokinase. Proc Natl Acad Sci USA 2015; 112: 11553-8. 